\title{
Association Between Serum miR-221-3p and Intravenous Immunoglobulin Resistance in Children With Kawasaki Disease
}

\section{Fengchuan Jing}

Chongqing Medical University Affiliated Children's Hospital

Haobo Weng

Chongqing Medical University Affiliated Children's Hospital

Jing Zhang

Chongqing Medical University Affiliated Children's Hospital

Qiongfei Pei

Chongqing Medical University Affiliated Children's Hospital

Ruixi Liu

Chongqing Medical University Affiliated Children's Hospital

Qijian Yi ( $\nabla$ qjyi2003@hotmail.com )

Chongqing Medical University Affiliated Children's Hospital https://orcid.org/0000-0002-4461-3904

\section{Research Article}

Keywords: Kawasaki disease, microRNA-221, intravenous immunoglobulin resistance

Posted Date: July 26th, 2021

DOI: https://doi.org/10.21203/rs.3.rs-735471/v1

License: (c) (1) This work is licensed under a Creative Commons Attribution 4.0 International License. Read Full License

Version of Record: A version of this preprint was published at Clinical and Experimental Medicine on January 18th, 2022. See the published version at https://doi.org/10.1007/s10238-021-00776-y. 


\section{Abstract}

Objectives

Intravenous immunoglobulin (IVIG) resistance was a major cause of coronary artery lesions in children with Kawasaki disease (KD). However, the cause of IVIG resistance in KD remains unknown. miR-221-3p has been confirmed involved in cardiovascular diseases and rheumatoid arthritis. The purpose of this study was to investigate the association between miR-221-3p and IVIG resistance in children with KD.

Methods

$55 \mathrm{KD}$ patients and 29 healthy controls (HCs) were enrolled in this study. KD patients were divided into group of sensitive to IVIG (IVIG-response, $n=42$ ) and group of resistant to IVIG (IVIG-resistance, $n=13$ ). Reverse transcription-quantitative polymerase chain reaction (RT-qPCR) were used to detect the levels of miR-221-3p.

Results

Compared with the HCs group, miR-221-3p were significantly increased in the KD group $(p<0.05)$, and the IVIG-resistance group had higher levels of miR-221-3p than those in the IVIG-response group $(p<0.05)$. CRP (C-reactive protein), PCT (procalcitonin), NLR (neutrophil-lymphocyte ratio) were positively correlated with miR-221-3p in KD patients. In addition, the group of IVIG resistance had a higher level of Kobayashi Score $(p<0.001)$. The receiver operating characteristic (ROC) curve showed that miR-221-3p had a better value for diagnosis IVIG resistance in children with KD than Kobayashi Score and the combination of both with the AUC of $0.811(95 \% \mathrm{Cl}, 0.672-0.951), 0.793(95 \% \mathrm{Cl}, 0.618-0.968)$ and $0.797(95 \% \mathrm{Cl}, 0.619-$ 0.974), respectively.

Conclusions

miR-221-3p might be involved in the pathogenesis of KD and IVIG resistance and miR-221-3p can be used as a new potential biomarker to predict of IVIG resistance in children with KD.

\section{What Is Known}

- IVIG resistance in KD was mainly caused by immune imbalance and inflammation, and miR-221-3p is involved in cardiovascular diseases and immune disease.

\section{What Is New}

- In this study, we have found the relationship between miR-221-3p and IVIG resistance in KD, and serum miR-221-3p can be used as a potential diagnostic biomarker to predict IVIG resistance in KD.

\section{Introduction}


Kawasaki disease (KD) is an acute self-limiting febrile vasculitis that mainly affects children under five years old [1]. Since its first discovery in 1960s, KD has been identified worldwide and-now become the leading cause of acquired heart disease in children in developed countries [2]. The most severe complication of KD is coronary artery lesions, which could ultimately lead to coronary heart disease (CHD) in young adults, resulting in myocardial ischemia, infarction, and even sudden death [35]. Although a single dose of $2 \mathrm{~g} / \mathrm{kg}$ intravenous immunoglobulin (IVIG) combined with aspirin regimen considered to be the standard treatment for KD, there is still approximately $20 \%$ of the cases are insensitive to IVIG, and around $10 \%$ of them develop into serious coronary artery abnormalities [6]. As a result, further research regarding the pathogenesis of IVIG resistance in children with KD is necessary.

miR-221, a member of antiangiogenic gene-regulating miRNAs family, is encoded by a co-transcribed cluster on the $\mathrm{X}$ chromosome and abundant distribution in endothelial and smooth muscle cells of human vessels [11]. Lots of studies have shown that microRNAs (miRNAs) are involved in pathogenesis of some diseases, such as cardiovascular diseases, inflammatory diseases, and immunologic diseases [7-10]. Pandis et al. found that the expression levels of miR-221 were significantly higher in synovial fibroblast (SF) in patients with rheumatoid arthritis (RA) [12]. Yang et al. demonstrated that downregulation of miR-221 can significantly inhibit the levels of TNF-a, IL-6, IL-1 $\beta$ and CXCL16 in fibroblast-like synoviocytes (FLS) cells in patients with RA [13]. Also, studies have found that miR-221 expression increases significantly in acute KD patients [14]. However, the relationship between miR-221 and IVIG resistance remains unclear. Thus, the purpose of this study is to investigate the role of miR-221$3 p$ on IVIG resistance in children with KD.

\section{Method}

\subsection{Study subjects}

Fifty-five KD patients (KD group) consisting of 37 males and 18 females (average age $2.648 \pm 1.832$ years) and twenty-nine healthy controls (HCs) children consisting of 19 males and 10 females (average age 3.191 \pm 1.075 years) were enrolled in this study from April to August of 2019 consecutively. All subjects met the criteria of American Heart Association (AHA) Scientific Statement. An IVIG nonresponder was defined by recrudescent or persistent fever at least 36 hours after the initial infusion of IVIG [2] and Kobayashi system [15] was used to predict IVIG resistance. This study was approved by the institutional ethics board of Children's Hospital of Chongqing Medical University; and written informed consents were obtained from all subjects or guardians.

\subsection{Sample Collection}

The blood samples were collected from the KD patients on the day of admission before given IVIG and aspirin and immediately centrifuged at $4^{\circ} \mathrm{C}$ for $10 \mathrm{~min}$ at 3,000 revolutions $/ \mathrm{min}$ to isolate the serum. The same procedures were conducted to the $\mathrm{HCs}$, and all serum samples were stored at $-80^{\circ} \mathrm{C}$. 
RNA was extracted using a miRNeasy serum/plasma kit (Qiagen, Hilden, Germany, Cat. No: 217184) according to the manufacturer's instructions. The primer of miR-221-3p was quantitated by reverse transcription and Real-Time qPCR analysis using Qiagen-based RT-qPCR assay (Qiagen, Hilden, Germany, Cat. No: 339320). The cycling conditions were $95^{\circ} \mathrm{C}$ for 2 min followed by 40 cycles at $95^{\circ} \mathrm{C}$ for $10 \mathrm{sec}$ and $56^{\circ} \mathrm{C}$ for $1 \mathrm{~min}$. All reactions were performed in triplicate. The expression was quantified using the $2^{-\Delta \Delta} \mathrm{Cq}$ values and hsa-miR-103a-3p served as a control for calibrate the expression levels of miR-221-3p.

\subsection{Statistical analysis}

Demographic and clinical characteristic of subjects were expressed as mean values and as total numbers according to the applied statistical methods. The distribution of these variables was evaluated with Shapiro-Wilk normality test. Normally distributed variables were compared using t-test. The differences levels of miR-221-3p in serum between KD patients and HCs were analyzed by $2^{-\Delta \Delta} \mathrm{Cq}$ method. The correlation of miR-221-3p with other clinical characteristics was assessed with Pearson correlation. The diagnostic accuracy of miR-221-3p were measured by the receiver-operating characteristic (ROC) curves and the area under the ROC curve (AUC). Reported $p$-values are two-sided and a $p$ value less 0.05 was considered the difference significantly. All analyses were performed by Statistical Package for Social Sciences for Windows 24.0 (SPSS Inc., Chicago, IL, USA).

\section{Results}

\subsection{The Expression of miR-221-3p in KD Patients and Healthy Controls}

Demographic features between KD group and HCs group were examined and there was no significant difference in age and gender between these two groups $(p \otimes 0.05)$. The qRT-PCR method was used to detect the expression of miR-221-3p in $55 \mathrm{KD}$ patients and 19 healthy controls. The results showed that, the level of serum miR-221-3p were significantly higher in KD patients than that of in healthy controls $(p=0.002$, Figures 1$)$.

\subsection{The Correlations between miR-221-3p and Clinical Characteristics in KD Patients}

Pearson correlation analysis showed that serum miR-221-3p was positively correlated with clinical inflammatory factors in all KD patients, including percentage of neutrophils (N\%), C-reactive protein (CRP), procalcitonin (PCT), and Neutrophil-Lymphocyte ratio (NLR) $(r=0.494, p<0.001 ; r=0.545, p<0.001 ; r$ $=0.399, p=0.568 ; r=0.436, p=0.001$, respectively $)$, and negative with percentage of lymphocyte $(\mathrm{L} \%) \otimes r$ $=-0.450, p=0.001 \otimes($ Table 1$)$.

Interestingly, serum miR-221-3p was significantly association with total dosage of IVIG and administration times of IVIG in KD group ( $r=0.501, p<0.001 ; r=0.558, p<0.001$, respectively), while no relationship with the onset age, gender, and weight (all $p>0.05$, Table 1 ).

\subsection{Clinical Characteristics in IVIG resistance group and IVIG response group.}


A total of 42 IVIG responders and 13 IVIG non-responders were enrolled in this study. IVIG non-responders were $(n=13)$ more likely to have a longer duration of fever, a higher total dosage of IVIG $(p<0.001)$, higher levels of mean platelet volume (MPV), platelet distribution width (PDW), neutrophils, total bilirubin (TLB), alanine transaminase (ALT), aspartate aminotransferase (AST), CRP, PCT, NLR, prothrombin and lower levels of lymphocyte, platelet count (PLT), and albumin (ALB) than that of IVIG responders group, while age, gender, weight and time point of IVIG were not different (Table 2).

\subsection{Expression of miR-221-3p and Score of Kobayashi System in IVIG resistance group and IVIG response group.}

Statistical tests revealed that the level of serum miR-221-3p was significantly higher in group of resistance to IVIG than that of group of IVIG responses (1.782 \pm 0.7 vs $1 \pm 0.552, p=0.002)$ (Figure 2). There was also a higher Kobayashi scores in KD patients with IVIG resistance $(p<0.001)$ (Table 3$)$.

\subsection{The ROC Curves Analysis of serum miR-221-3p in predicting IVIG resistance in children with KD}

Receiver operating characteristic (ROC) curve analysis was used to explore the usefulness of serum miR221-3p, Kobayashi Score and combination of both to predict unresponsiveness to IVIG in children with $K D$. The results showed that serum miR-221-3p reflected obvious separation between group of IVIG resistance and group of IVIG response; the AUC was $0.811(95 \% \mathrm{Cl}, 0.672-0.951$, $p=0.001), 0.793(95 \% \mathrm{Cl}, 0.618-0.968, p=0.002)$ and $0.797(95 \% \mathrm{Cl}, 0.619-0.974, p=0.001)$, respectively (Figure 3).

\section{Discussion}

The characterization of KD is an inflammatory in all the medium-sized arteries, especially coronary artery lesions (CALs). Accumulating studies reported that $10 \%$ to $20 \%$ of KD patients' unresponsiveness to initial treatment with IVIG, whom had increased risk to develop of CALs [16, 17]. Early identification of IVIG resistance may be important to reduce CALs in children with KD. However, the pathogenesis of nonresponsiveness of IVIG in children with KD remains unknown.

Total number of $55 \mathrm{KD}$ patients were enrolled in this study, out of which 13 of them (23.6\%) were diagnosed with IVIG resistance according to the 2017 American Heart Association guidelines. Our observation showed that patients with IVIG nonresponsiveness have a longer duration of fever, a higher total dosage of IVIG and significantly higher levels of CRP, N\%, PCT, NLR, ALT and AST than that of group of IVIG responders, and coincident with other reports [18-20]. The IVIG resistance group also showed lower levels of lymphocyte, PLT, albumin and sodium concentrations than that of IVIG responding group. Up to now, these laboratory data have been used as predictors of the IVIG non-responders [15, 21-24]. These results may collectively reflect the presence of severe vascular inflammation in children with KD and result in IVIG resistance. 
Evidences have shown that the inflammatory cytokines and chemokines such as TNF- $a$, IL-1, IL-6, and ICAM-1, are excessively increased during the acute febrile phase, indicating immune activation may contribute to the pathogenesis of KD [25,26]. Recently, numerous studies have implicated that Toll-like receptors (TLRs) signaling pathway in inflammatory vascular pathological conditions may play an important role in development of KD [27-30]. Our previous study clarified that TLR4/NF-KB signaling pathway was involved in the process of coronary artery lesions. These results may suggest that regulating expression of TLRs will protect children with KD from coronary artery lesions [31]. miR-221-3p, one of the miRNAs that is conserved among vertebrates, was detected in immunologic and inflammatory disease as well as cardiovascular disease. Evidence has shown that miR-221-3p could be a regulator of TLRs [13]. Regulation of miR-221-3p can affect the level of pro-inflammatory cytokines, such as TNF-a, IL$1 \beta$ and IL-6, in FLS cells when stimulated by LPS [32]. Up regulation of miR-221-3p in TLR4 can enhance the secretion of IL- 6 and IL-8, and down regulation the level of anti-inflammatory factors of CXCL13 and IL-10 [33]. Consistent results were also observed that there was relationship between miR-221-3p and expression of IL-6, TNF-a and activation of NF-kB and MAPKs in inflammatory lung disorder [34].

In our study, we found that serum miR-221-3p was elevated in children with acute phrase of KD, which is consistent with previous study [14]. For the first time, we reported that serum miR-221-3p was extremely higher in group of IVIG non-responders than that of group of IVIG responders. The-serum miR-221-3p levels have significantly correlated with many key inflammatory indexes, such as N\%, CRP, PCT, NLR and even total dosage of IVIG and given times of IVIG in KD patients. These results elicited that miR-221-3p may exhibit an important role in initiating inflammatory reaction involved in pathogenesis of KD and IVIG resistance.

Interleukin-6 (IL-6) is one of the pro-inflammatory with pleiotropic activity. It is released by not only T cells and macrophages, but also vascular endothelial cells, fibroblasts, and many other cells in response to various stimulation including TLR ligands and proinflammatory cytokines such as TNF-a or IL-1 [35]. It also has correlation with the acute phase of KD and IVIG non-responsiveness [19]. SOCS3 is a member of the IL-6 signaling family, it directly interacts with Janus kinase (JAK) signal transducer and inhibits of transcription 3 (STAT3) pathway and regulates immune reaction, cell growth and differentiation. According to a recent study via bioinformatics analysis and dual luciferase reporter gene assays, SOCS3 was found to be the target gene of miR-221-3p in hepatocellular carcinoma (HCC), which demonstrated that regulating the miR-221-3p/SOCS3 axis may affect the JAK/STAT signaling pathway and ultimately alter HCC tumor growth [36, 37 ]. Furthermore, Chatterjee PK et al. [38] had reported that IL-6/STAT3 pathway may be an underlying mechanism of inflammation in vascular endothelial. Whether or not miR$221-3 p / S O C S 3$ axis is involved in the development of KD and IVIG resistance need further study.

Kobayashi score (KS) has been used to predict CALs and IVIG resistance. The items of the KS scores included age in months, day of illness at initial treatment, N\%, PLT, AST, sodium, and CRP to generate a scoring model, and had a specificity of $68 \%$ and a sensitivity of $86 \%$. Here, we have shown that serum miR-221-3p had a better value than the Kobayashi system to predict IVIG non-responsivenss with the AUC of 0.811 by ROC analysis. 


\section{Conclusions}

In conclusion, our study showed that KD patients with IVIG resistance had a higher level of miR-221-3p and a better value than Kobayashi score to predict IVIG resistance, miR-221-3p may be used as a proinflammatory factor and a potential biomarker of IVIG resistance in KD and provide a novel mechanism and a new therapeutic target for KD.

\section{Declarations}

\section{Study limitations.}

There are limitations in this study. Firstly, the sample size was relatively small and it was finished in a single center. Secondly, we did not collect serum samples from KD patients after being treated with IVIG in subacute and convalescent.

\section{Funding}

This work was supported by the Outstanding Young Talent Training Program Class B of Children's Hospital Affiliated to Chongqing Medical University; Award Number: None; Recipient: DR. Ruixi Liu.

\section{Conflicts of interest}

The authors reported no conflict of interest.

\section{Data Availability}

All datasets used or analyzed during the current study are available from the corresponding author on reasonable request.

\section{Code availability}

Not applicable.

\section{Authors' contributions}

Fengchuan Jing has a substantial contribution to the conception and design of this study, acquisition of data, analysis of data and draft the article.

Haobo Weng contributes to acquisition of data, analysis of data and interpretation of data.

Qiongfei Pei contributes to interpretation of data.

Jing Zhang contributes to interpretation of data.

Ruixi Liu * has a substantial to design of the study and revise the article. 
Qijian $\mathrm{Yi}^{*}$ has a substantial contribution to revise the article critically for important intellectual content and final approval of the version to be published.

All authors approved the final manuscript as submitted.

\section{Ethical approval}

This study was approved by the institutional ethics board of Children's Hospital of Chongqing Medical University.

\section{Consent to participate:}

Informed consent was obtained from all subjects or guardians.

\section{Consent for publication}

All authors approved the final manuscript as submitted.

\section{References}

1. Newburger JW, Takahashi M, Gerber MA, et al. Diagnosis, treatment, and longterm management of Kawasaki disease: a statement for health professionals from the Committee on Rheumatic Fever, Endocarditis and Kawasaki Disease, Council on Cardiovascular Disease in the Young, American Heart Association. Pediatrics, 2004, 114(6):1708-33.

2. Mccrindle BW, Rowley AH, Newburger JW, et al. Diagnosis, Treatment, and Long-Term Management of Kawasaki Disease: A Scientific Statement for Health Professionals From the American Heart Association. Circulation, 2017, 135(17): e927-e999.

3. Daniels LB, Tjajadi MS, Walford HH, et al. Prevalence of Kawasaki disease in young adults with suspected myocardial ischemia. Circulation, 2012, 125(20):2447-53.

4. Hayasaka S, Nakamura Y, Yashiro M, et al. Analyses of fetal cases of Kawasaki disease in Japan using vital statistical data of 27 years. J Epidemiol, 2003, 13(5): 246-250.

5. Lin MT, Sun LC, Wu ET, Wang JK, Lue HC, Wu MH. Acute and late coronary outcomes in 1073 patients with Kawasaki disease with and without intravenous $\gamma$-immunoglobulin therapy. Arch Dis Child, 2015, 100(6):542-7.

6. Marc OJ, Shulman ST, Fox LM, et al. Three Linked Vasculopathic Processes Characterize Kawasaki Disease: A Light and Transmission Electron Microscopic Study. PLoS ONE, 2012, 7(6): e38998

7. Bartel DP. MicroRNAs: target recognition and regulatory functions. Cell,2009, 136(2):215-33.

8. Small EM, Olson EN. Pervasive roles of microRNAs in cardiovascular biology. Nature, 2011, 469(7330):336-42.

9. Zhang X, Shao S, Geng H, et al. Expression profiles of six circulating microRNAs critical to atherosclerosis in patients with subclinical hypothyroidism: a clinical study. J Clin Endocrinol 
Metab,2014, 99(5): E766-74.

10. O'Connell RM, Rao DS, Chaudhuri AA, Baltimore D. Physiological and pathological roles for microRNAs in the immune system. Nat Rev Immunol, 2010, 10(2):111-22.

11. Corsten MF, Heggermont W, Papageorgiou AP, et al. The microRNA-221/-222 cluster balances the antiviral and inflammatory response in viral myocarditis. Eur Heart J. 2015, 36(42):2909-19.

12. Pandis I, Ospelt C, Karagianni N, et al. Identification of microRNA-221/222 and microRNA-323-3p association with rheumatoid arthritis via predictions using the human tumour necrosis factor transgenic mouse model. Ann Rheum Dis, 2012, 71(10):1716-23.

13. Yang S, Yang Y. Downregulation of microRNA221 decreases migration and invasion in fibroblast-like synoviocytes in rheumatoid arthritis. Mol Med Rep, 2015, 12(2):2395-401.

14. Rong X, Jia L, Hong L, et al. Serum miR-92a-3p as a New Potential Biomarker for Diagnosis of Kawasaki Disease with Coronary Artery Lesions. Journal of Cardiovascular Translational Research, 2017, 10(1):1-8.

15. Kobayashi T, Inoue Y, Takeuchi K, et al. Prediction of intravenous immunoglobulin unresponsiveness in patients with Kawasaki disease. Circulation, 2006, 113(22):2606-12.

16. Schiller B, Elinder G. Inflammatory parameters and soluble cell adhesion molecules in Swedish children with Kawasaki disease relationship to cardiac lesions and intravenous immunoglobulin treatment. Acta Paediatr, 1999, 88(8):844-8

17. Burns JC, Capparelli EV, Brown JA, Newburger JW, Glode MP. Intravenous gamma-globulin treatment and retreatment in Kawasaki disease. US/Canadian Kawasaki Syndrome Study Group. Pediatr Infect Dis J, 1998, 17(12):1144-8.

18. Uehara R, Belay ED, Maddox RA, et al. Analysis of potential risk factors associated with nonresponse to initial intravenous immunoglobulin treatment among Kawasaki disease patients in Japan. Pediatr Infect Dis J. 2008; 27(2):155-60.

19. Sato S, Kawashima H, Kashiwagi Y, Hoshika A. Inflammatory cytokines as predictors of resistance to intravenous immunoglobulin therapy in Kawasaki disease patients. International Journal of Rheumatic Diseases, 2013, 16(2):168-172.

20. Shao S, Luo C, Zhou K, Hua Y, Wang. Predictive value of serum procalcitonin for both initial and repeated immunoglobulin resistance in Kawasaki disease: a prospective cohort study. Pediatric Rheumatology, 2019, 17(1):78.

21. Sano T, Kurotobi S, Matsuzaki K, et al. Prediction of non-responsiveness to standard high-dose gamma-globulin therapy in patients with acute Kawasaki disease before starting initial treatment. Eur J Pediatr, 2007, 166(2):131-137.

22. Egami K, Muta $\mathrm{H}$, Ishii M, et al. Prediction of resistance to intravenous immunoglobulin treatment in patients with Kawasaki disease. J Pediatr, 2006, 149(2):237-240.

23. Tremoulet $A H$, Best BM, Song $S$, et al. Resistance to intravenous immunoglobulin in children with Kawasaki disease. J Pediatr, 2008, 153(1): 117-121.e3. 
24. Fu PP, Du ZD, Pan YS. Novel predictors of intravenous immunoglobulin resistance in Chinese children with Kawasaki disease. Pediatr Infect Dis, 2013, 32(8):319-23.

25. Furukawa S, Matsubara T, Yone K, Hirano Y, Okumura K, Yabuta K. Kawasaki disease differs from anaphylactoid purpura and measles with regard to tumour necrosis factor-a and interleukin 6 serum. Eur J Pediatr, 1992, 151(1):44-47.

26. Furukawa S, Imai K, Matsubara T, Yone K, Yabuta Ket. Increased levels of circulating intercellular adhesion molecule 1 in Kawasaki disease. Arthritis Rheumatology, 2014, 35(6):672-677.

27. Hui-Yuen JS, Duong TT, Yeung R. TNF-alpha is necessary for induction of coronary artery inflammation and aneurysm formation in an animal model of Kawasaki disease. Journal of Immunology, 2006, 176(10):6294-6301.

28. Lee $Y$, Schulte $D$ J, Shimada K, et al. Interleukin-1 $\beta$ Is Crucial for the Induction of Coronary Artery Inflammation in a Mouse Model of Kawasaki Disease. Circulation, 2012, 125(12):1542-1550.

29. Rosenkranz ME, Schulte DJ, Agle L, et al. TLR2 and MyD88 Contribute to Lactobacillus casei ExtractInduced Focal Coronary Arteritis in a Mouse Model of Kawasaki Disease. Circulation, 2005, 112(19):2966-2973.

30. Armaroli G, Verweyen E, Pretzer C, et al. S100A12-induced sterile inflammatory activation of human coronary artery endothelial cells is driven by monocyte-derived interleukin $1 \mathrm{~b}$ : implications for Kawasaki disease pathology. Arthritis and Rheumatology, 2018, 71(5).

31. Gao F, Si F, Feng S, Yi Q, Liu R. Resistin Enhances Inflammatory Cytokine Production in Coronary Artery Tissues by Activating the NF-KB Signaling. Biomed Res Int, 2016, 2016 (3):1-8.

32. Song J, Ouyang Y, Che J, et al. Potential value of miR-221/222 as diagnostic, prognostic, and therapeutic biomarkers for diseases. Front Immunol, 2017, 8(3)

33. Quero L, Tiaden AN, Hanser E, et al. miR-221-3p Drives the Shift of M2-Macrophages to a ProInflammatory Function by Suppressing JAK3/STAT3 Activation. Front Immunol, 2020, 10:3087.

34. Zhao D, Zhuang N, Ding Y, Kang Y, Shi L. miR-221 activates the NF-kB pathway by targeting A20. Biochemical and Biophysical Research Communications, 2016, 472(1):11-18.

35. Kang S, Tanaka T, Narazaki M, Kishimoto T. Targeting Interleukin-6 Signaling in Clinic. Immunity, 2019, 50(4):1007-1023.

36. Xu G, Yang F, Ding CL, et al. MiR-221 accentuates IFNs anti-HCV effect by downregulating SOCS1 and SOCS3. Virology, 2014, 462-463(1):343-350.

37. Li H, Zhang B, Ding M, et al. C1QTNF1-AS1 regulates the occurrence and development of hepatocellular carcinoma by regulating miR-221-3p/ SOCS3. Hepatology International, 2019, 13(12).

38. Chatterjee PK, Al-Abed Y, Sherry B, Metz CN. Cholinergic agonists regulate JAK2/STAT3 signaling to suppress endothelial cell activation. Am J Physiol. 2009;297:C1294-306.

\section{Tables}

Table 1. Correlations between serum miR-221-3p and clinical characteristics in all KD patients. 


\begin{tabular}{|c|c|c|}
\hline \multirow[t]{2}{*}{ Variables } & \multicolumn{2}{|c|}{ Serum Levels of miR-221-3p } \\
\hline & $r$ & $p$ \\
\hline \multicolumn{3}{|l|}{ Demographics } \\
\hline Age & 0.196 & 0.151 \\
\hline Weight & 0.122 & 0.375 \\
\hline Time point of IVIG (day) & -0.224 & 0.122 \\
\hline Total dosage of IVIG (g) & 0.501 & $<0.001^{\star \star \star}$ \\
\hline Given times of IVIG & 0.558 & $<0.001 * \star \star$ \\
\hline \multicolumn{3}{|l|}{ Blood test } \\
\hline White blood cell $\left(10^{9} / \mathrm{L}\right)$ & 0.133 & 0.334 \\
\hline Platelet count $\left(10^{9} / \mathrm{L}\right)$ & -0.242 & 0.075 \\
\hline Red blood cell count $\left(10^{12} / \mathrm{L}\right)$ & 0.239 & 0.079 \\
\hline Hemoglobin (g/l) & -0.05 & 0.715 \\
\hline Percentage of neutrophil (\%) & 0.494 & $<0.001^{\star \star \star *}$ \\
\hline Percentage of lymphocyte (\%) & -0.450 & $0.001 * \star \star$ \\
\hline \multicolumn{3}{|l|}{ Biochemical test } \\
\hline Alanine transaminase (IU/L) & 0.226 & 0.097 \\
\hline Aspartate aminotransferase (IU/L) & 0.068 & 0.621 \\
\hline Albumin $(g / L)$ & -0.237 & 0.082 \\
\hline \multicolumn{3}{|l|}{ Inflammatory factor } \\
\hline C-reactive protein (mg/L) & 0.545 & $<0.001^{\star \star \star *}$ \\
\hline Erythrocyte sedimentation rate (mm/L) & 0.125 & 0.362 \\
\hline Procalcitonin (ng/ml) & 0.568 & $<0.001 * \star \star$ \\
\hline Neutrophil/Lymphocyte ratio & 0.47 & $0.001^{\star \star \star}$ \\
\hline Platelet/Lymphocyte ratio & 0.231 & 0.09 \\
\hline \multicolumn{3}{|l|}{ Coagulation Function } \\
\hline Prothrombin (second) & 0.193 & 0.174 \\
\hline Activated partial thromboplastin time (second) & 0.111 & 0.436 \\
\hline Fibrinogen $(\mathrm{g} / \mathrm{L})$ & 0.178 & 0.212 \\
\hline
\end{tabular}




\begin{tabular}{|lll|}
\hline Thrombin time (second) & 0.106 & 0.46 \\
\hline D-dimer $(\mathrm{mg} / \mathrm{L})$ & 0.066 & 0.649 \\
\hline
\end{tabular}

$\star \star \star p<0.005$

Table 2. Laboratory values between IVIG Response and IVIG Resistance. 
Variables

IVIG Response

IVIG Resistance

$p$ value

$(n=42) \quad(n=13)$

\section{Demographics}

Age (y)

$2.379 \pm 1.748$

$2.349 \pm 1.211$

0.944

Gender (male/female)

$27 / 15$

$10 / 3$

0.51

Weight $(\mathrm{kg})$

$12.719 \pm 5.284$

$12.192 \pm 2.803$

0.643

Duration of fever (d)

$6.55 \pm 1.468$

$8.46 \pm 2.847$

$0.002^{\star \star \star}$

Usage of Intravenous Immunoglobulin

\begin{tabular}{llll}
\hline Time point of IVIG (day) & $6.3 \pm 1.493$ & $5.69 \pm 2.213$ & 0.929 \\
\hline Total dosage of IVIG $(\mathrm{g})$ & $25.357 \pm 7.1902$ & $43.269 \pm 18.3821$ & $p<0.001^{\star \star \star}$ \\
\hline Given times of IVIG & $1 \pm 0$ & $1.69 \pm 0.48$ & $p<0.001^{\star \star \star}$ \\
\hline Blood test & & & \\
\hline White blood cell $\left(10^{9} / \mathrm{L}\right)$ & $14.054 \pm 4.894$ & $17.707 \pm 8.732$ & 0.06 \\
\hline Red blood cell count $\left(10^{12} / \mathrm{L}\right)$ & $4.271 \pm 1.111$ & $4.342 \pm 1.37$ & 0.833 \\
\hline Hemoglobin $(\mathrm{g} / \mathrm{I})$ & $108.3 \pm 14.659$ & $102.8 \pm 11.003$ & 0.867 \\
\hline Platelet count $\left(10^{9} / \mathrm{L}\right)$ & $411.02 \pm 129.758$ & $304.77 \pm 128.486$ & $0.017^{\star}$ \\
\hline Mean platelet volume(fL) & $10.129 \pm 1.202$ & $11.177 \pm 1.044$ & $0.006^{\star \star}$ \\
\hline Platelet distribution width(fL) & $11.567 \pm 2.248$ & $13.792 \pm 2.282$ & $0.006^{\star *}$ \\
\hline Neutrophil count $\left(10^{9} / \mathrm{L}\right)$ & $0.626 \pm 0.141$ & $0.799 \pm 0.136$ & $0.001^{\star \star *}$ \\
\hline Lymphocyte count $\left(10^{9} / \mathrm{L}\right)$ & $0.309 \pm 0.125$ & $0.159 \pm 0.121$ & $0.001^{\star \star *}$ \\
\hline
\end{tabular}

\section{Biochemical test}

\begin{tabular}{llll}
\hline Total bilirubin(umol/L) & $5.117 \pm 2.574$ & $16.138 \pm 18.873$ & $p<0.001^{\star \star \star}$ \\
\hline Alanine transaminase(IU/L) & $29.095 \pm 31.393$ & $92.754 \pm 107.538$ & $0.001^{\star \star \star}$ \\
\hline Aspartate aminotransferase (IU/L) & $29.181 \pm 10.245$ & $69.331 \pm 107.585$ & $0.018^{\star}$ \\
\hline Albumin (g/L) & $37.855 \pm 4.186$ & $33.231 \pm 4.059$ & $0.002^{\star \star \star}$ \\
\hline Sodium & $137.393 \pm 2.609$ & $134.6 \pm 2.9439$ & $0.002^{\star \star \star}$ \\
\hline Inflammatory factor & & & \\
\hline C-reactive protein $(\mathrm{mg} / \mathrm{L})$ & $52.44 \pm 33.023$ & $101.46 \pm 62.89$ & $0.001^{\star \star \star}$ \\
\hline
\end{tabular}




\begin{tabular}{llll} 
Erythrocyte sedimentation rate $(\mathrm{mm} / \mathrm{L})$ & $75.62 \pm 24.528$ & $81.38 \pm 32.577$ & 0.564 \\
\hline Procalcitonin $(\mathrm{ng} / \mathrm{ml})$ & $0.644 \pm 0.81$ & $5.683 \pm 5.197$ & $p<0.001^{\star \star *}$ \\
\hline Neutrophil/Lymphocyte ratio & $3.7698 \pm 6.936$ & $11.348 \pm 9.31$ & $0.003^{\star \star *}$ \\
\hline Platelet/Lymphocyte ratio & $179.563 \pm 338.748$ & $270.5322 \pm 327.808$ & 0.397
\end{tabular}

\section{Coagulation Function}

\begin{tabular}{llll}
\hline Prothrombin (second) & $12.335 \pm 0.917$ & $13.182 \pm 0.929$ & $0.016^{*}$ \\
\hline $\begin{array}{l}\text { Activated partial thromboplastin time } \\
\text { (second) }\end{array}$ & $30.068 \pm 2.746$ & $31.118 \pm 3.922$ & 0.419 \\
\hline Fibrinogen (g/L) & $6.01 \pm 1.35$ & $6.584 \pm 1.167$ & 0.179 \\
\hline Thrombin time (second) & $15.075 \pm 0.74$ & $14.945 \pm 0.906$ & 0.67 \\
\hline D-dimer (mg/L) & $2.13 \pm 3.853$ & $4.364 \pm 3.835$ & 0.107 \\
\hline
\end{tabular}

${ }^{*} p<0.05,{ }^{* \star} p<0.01,{ }^{* \star *} p<0.005$

Table 3. Kobayashi System in IVIG Response and IVIG Resistance.

\begin{tabular}{llll} 
Variables & $\begin{array}{l}\text { IVIG Response } \\
(\mathbf{n}=\mathbf{4 2})\end{array}$ & $\begin{array}{l}\text { IVIG Resistance } \\
(\mathbf{n}=\mathbf{1 3})\end{array}$ & $\boldsymbol{p}$ value \\
\hline Serum sodium (mmol/L) & $137.393 \pm 2.609$ & $134.6 \pm 2.944$ & $0.002^{\star \star \star}$ \\
\hline Aspartate aminotransferase (IU/L) & $29.181 \pm 10.244$ & $69.331 \pm 107.585$ & $0.018^{\star}$ \\
\hline Percentage of neutrophil & $0.626 \pm 0.141$ & $0.799 \pm 0.16$ & $0.001^{\star \star \star}$ \\
\hline Time point of IVIG (day) & $6.3 \pm 1.493$ & $5.69 \pm 2.213$ & 0.929 \\
\hline C-reactive protein $(\mathrm{mg} / \mathrm{L})$ & $52.44 \pm 33.023$ & $101.46 \pm 62.89$ & $0.001^{\star * \star}$ \\
\hline Platelet count $\left(10^{9} / \mathrm{L}\right)$ & $411.02 \pm 129.758$ & $304.77 \pm 128.486$ & $0.017^{\star}$ \\
\hline Age $(\mathrm{y})$ & $2.379 \pm 1.748$ & $2.349 \pm 1.211$ & 0.944 \\
\hline Kobayashi Score & $0.79 \pm 1.048$ & $3.54 \pm 2.696$ & $p<0.001^{\star \star \star}$
\end{tabular}

${ }^{\star} p<0.05, * \star \star p<0.005$

\section{Figures}




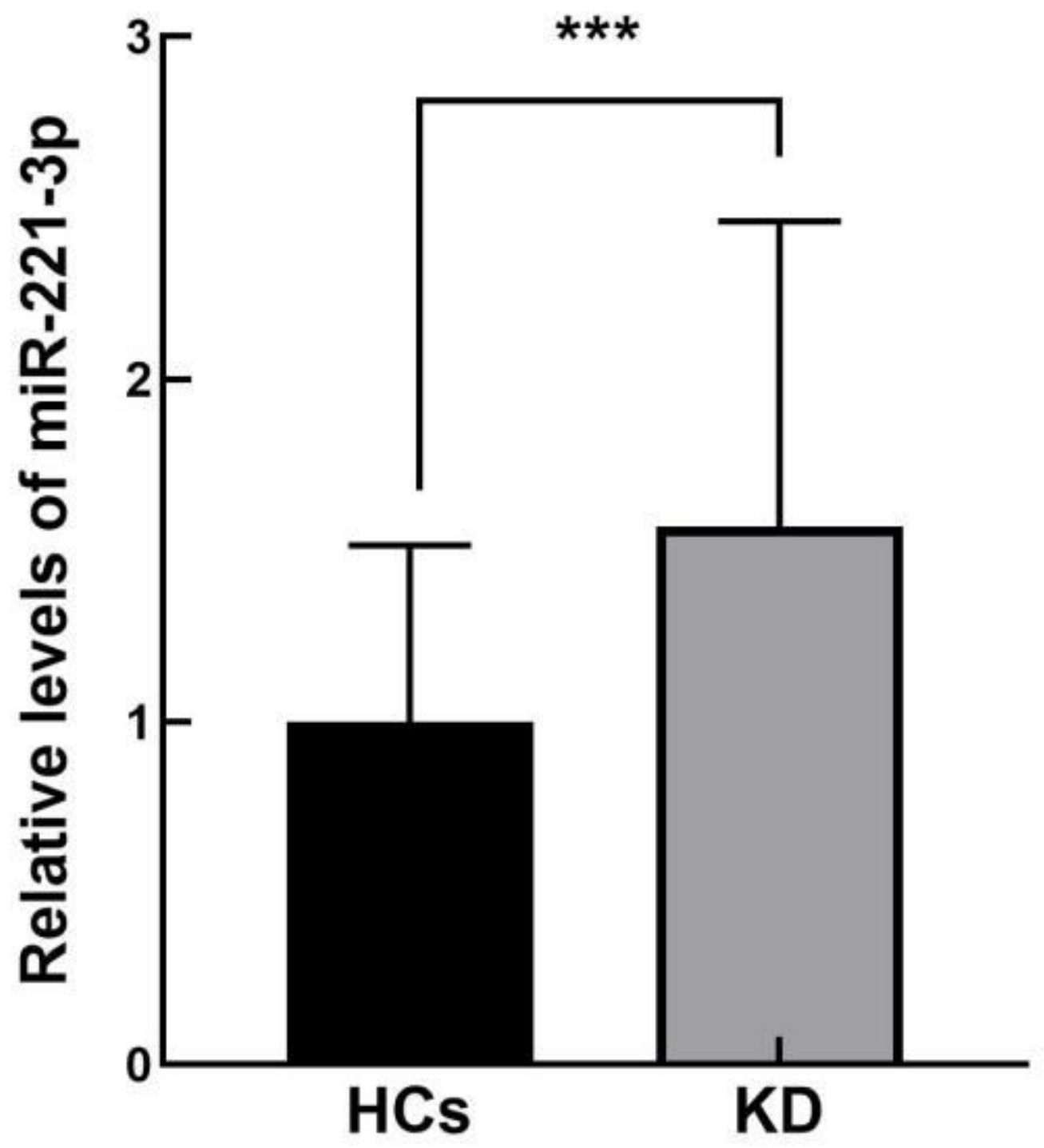

Figure 1

Serum levels of miR-221-3p in Kawasaki disease (KD) and healthy controls (HCs). ${ }^{\star \star \star} p<0.005$. 


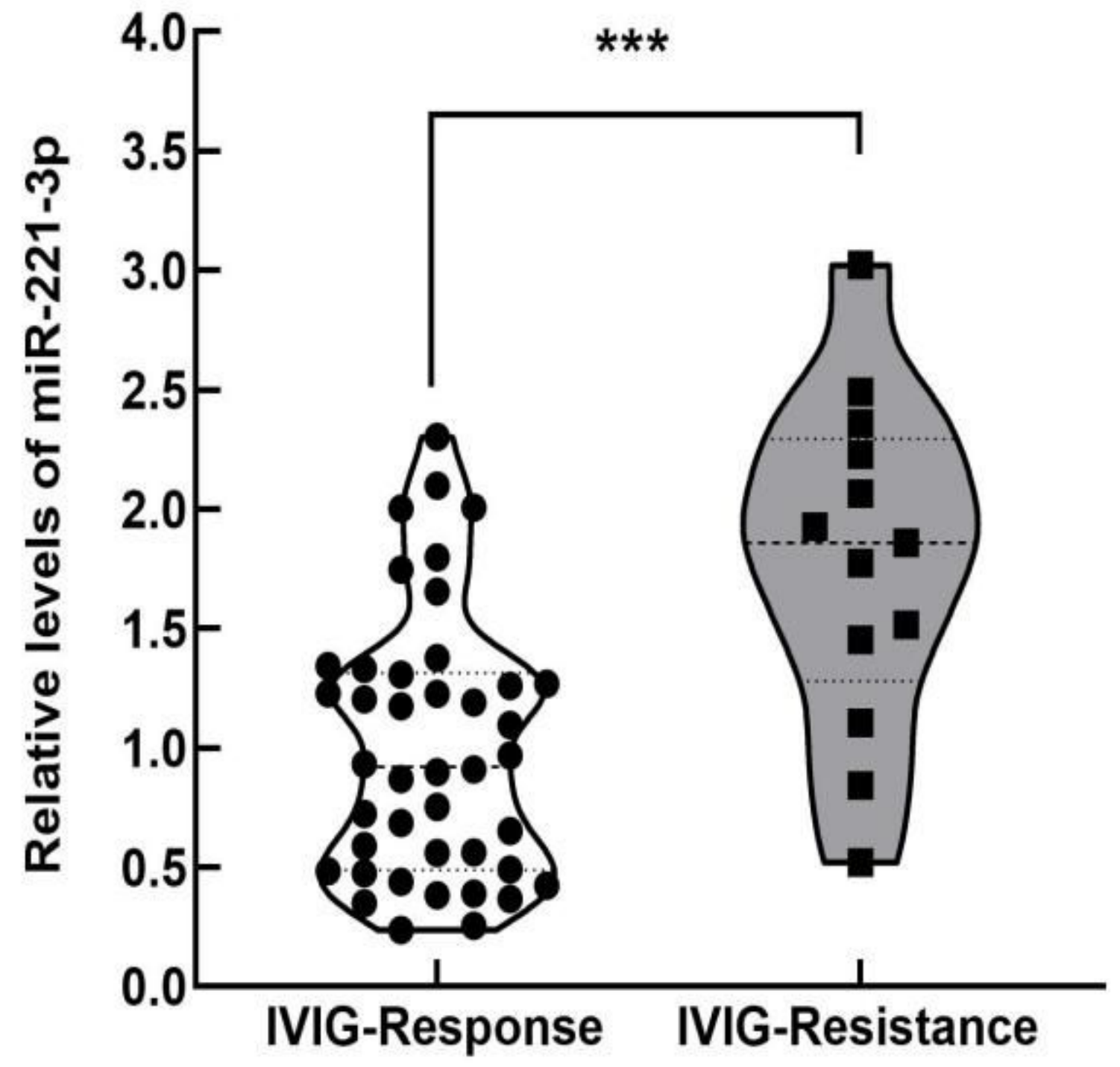

Figure 2

Serum levels of miR-221-3p in KD patients with IVIG Response and IVIG Resistance. ${ }^{\star \star \star} p<0.005$. 


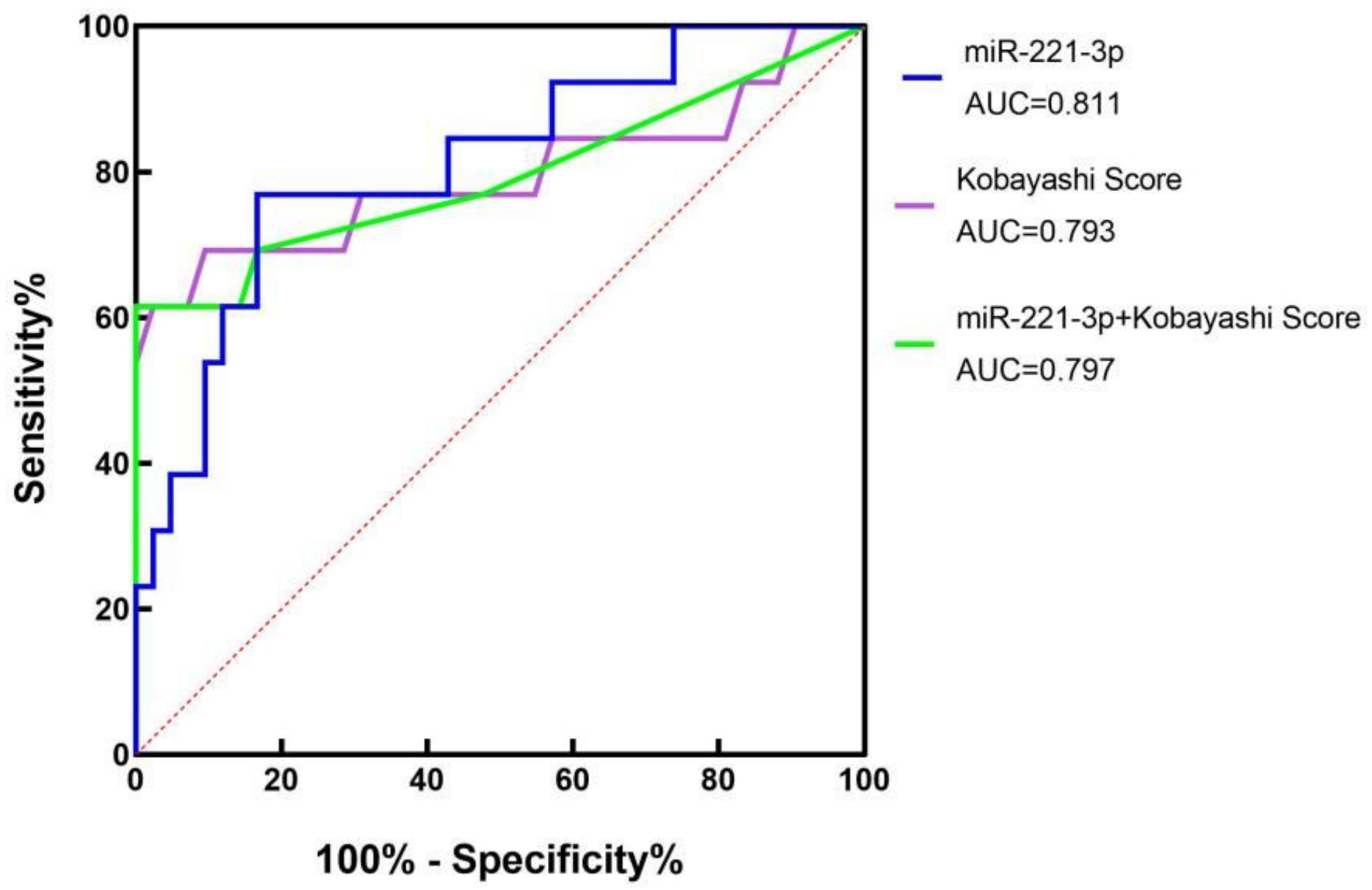

Figure 3

ROCs of various scores used to diagnosis KD patients with intravenous immunoglobulin (IVIG) resistance. Area under the curves (AUC): miR-221-3p (blue) $=0.811(95 \% \mathrm{Cl}, 0.672-0.951, p=0.001)$, Kobayashi Score (purple) $=0.793(95 \% \mathrm{Cl}, 0.618-0.968, \mathrm{p}=0.002)$ and combination of these two (green) parameters $=0.797(95 \% \mathrm{Cl}, 0.619-0.974, \mathrm{p}=0.001)$. 